\title{
Influences on the direction probabilities for the direction instability phenomenon in fiber ring lasers
}

\author{
M. A. Arshad $^{1,2}$, Arni Pratiwi ${ }^{1,2}$, A. Hartung $^{1}$, M. Jäger $^{1}$ \\ 1. Leibniz-Institute of Photonic Technology, Albert-Einstein-Straße 9, 07747 Jena, Germany \\ 2. Friedrich Schiller University, Faculty of Physics and Astronomy, Max-Wien-Platz 1, 07743 Jena, Germany
}

Recently, we observed a direction instability phenomenon, the spontaneous transition from two emission directions to to one emission direction, in a km-long fiber ring laser [1]. The setup in use is shown in Fig. 1. The cavity is pumped by a pump diode emitting at a wavelength of $976 \mathrm{~nm}$. The pump power enters the ring cavity by a pump signal combiner and reaches a $6 \mathrm{~m}$ long $\mathrm{Yb}$-doped active fiber section. Here, without any wavelength selective filter applied, a laser signal at around $1100 \mathrm{~nm}$ is emitted. The active fiber is followed by a long $(\sim \mathrm{km})$ passive fiber (Corning HI1060). Finally, an output tap coupler with a very low nominal coupling ratio of $0.01 \%$ at $1060 \mathrm{~nm}$ feeds most of the signal back to the pump signal combiner where it passes through the ring again, and a small part of the signal out of the cavity. As no optical isolator is included in the ring cavity clockwise and counter clockwise signals are allowed to propagate and induced at the laser threshold.

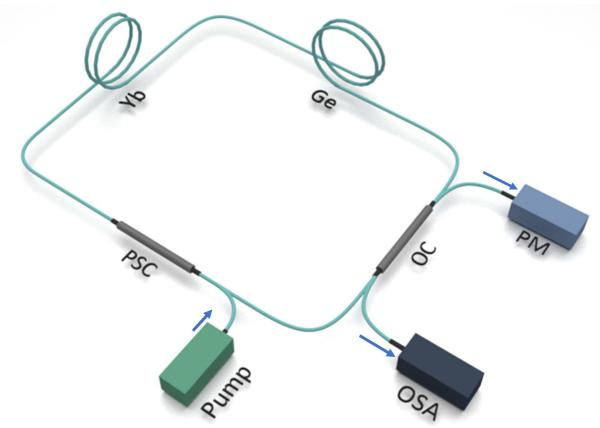

Fig. 1 General scheme of the all-fiber ring laser. The setup consists of a pump diode (pump), pump signal combiner (PSC), an active $\mathrm{Yb}$-doped fiber (Yb), a passive Ge-doped fiber (Ge), and an output coupler (OC).

This cavity shows the effect of spontaneous unidirectional lasing in combination with optical bistability. Unidirectional lasing occurs far above the laser threshold for up to now unknown reasons. The final direction is not predefined. The cavity can finally switch in any of the two directions. We observed that the direction probabilities can be significantly altered by the loss sequence of the passive fiber. We composed the passive fiber by $1 \mathrm{~km}$ long sections of two different fibers. Both fibers are Corning's HI 1060 fiber, share the same data sheet, and meet its requirements at the design wavelength of $1060 \mathrm{~nm}$. Nevertheless, the spectral dependence is locally different due to variations in the $\mathrm{OH}$ content.

We tested different fiber (and hence loss) sequences of two to four kilometer long cavities. Based on samples of 30 runs for each cavity layout we observed the following trends regarding the direction probabilities. The most equal probabilities were observed for an all-high-loss cavity with $2 \mathrm{~km}$ length. Here, one direction was triggered $37 \%$ of all runs and the other direction $67 \%$ of all runs. In contrast, including the low-loss kilometer seems to enforce a final direction with probabilities up to $100 \%$. In these cases the preferred final direction, starting at the active fiber, passes the high loss fiber first and the low loss fiber thereafter. Flipping the low-loss and the high-loss fiber also flipped the preference of the final direction. Passing the high linear loss fiber first might correspond with reducing the power and hence the nonlinear loss experienced by the laser wavelength. This indicates that nonlinear loss could have a crucial contribution to this new type of instability.

In order to achieve equal probabilities in both directions we set up a component-wise symmetric cavity where the output coupler is centered between two $1 \mathrm{~km}$ long identical passive fiber sections. Surprisingly, there is still a strong preference with one final direction being triggered $93 \%$ of the times. Only minor differences are left, to explain this imbalance all of them typically considered negligible regarding roundtrip loss like varying splice losses in the range of $0.2 \mathrm{~dB}$. This raises the question how much or how little imbalance is needed to influence the direction probabilities.

\section{References}

[1] M. A. Arshad, A. Hartung, and M. Jäger, "A stimulated Stokes Raman scattering-based approach for continuous wave supercontinuum generation in optical fibers," Laser Phys. Lett., vol. 16, no. 3, 2019, doi: 10.1088/1612-202X/aaff53. 